\title{
Changing Characteristics of Jupiter's Little Red Spot
}

\author{
A. F. Cheng ${ }^{1,9^{*}}$, A. A. Simon-Miller ${ }^{2}$, H. A. Weaver ${ }^{1}$, K. H. Baines ${ }^{3}$, G. S. Orton ${ }^{3}$, P. A. \\ Yanamandra-Fisher ${ }^{3}$, O. Mousis ${ }^{4}$, E. Pantin ${ }^{5}$, L. Vanzi ${ }^{6}$, L. N. Fletcher ${ }^{7}$, J. R. Spencer ${ }^{8}$, S. A. \\ Stern $^{9}$, J. T. Clarke ${ }^{10}$, M. J. Mutchler ${ }^{11}$, K. S. Noll ${ }^{11}$ \\ ${ }^{1}$ Johns Hopkins Applied Physics Laboratory, 11100 Johns Hopkins Rd., Laurel, MD 20723, \\ USA. \\ ${ }^{2}$ NASA/GSFC, Code 693, Greenbelt, MD 20771, USA \\ 3 Jet Propulsion Laboratory, 4800 Oak Grove Dr., Pasadena, Calif. 91109, USA \\ ${ }^{4}$ Institut UTINAM, CNRS-UMR 6213, Université de Franche-Comté, France \\ ${ }^{5}$ Centre d'Etudes Atomique CEA/SAp, Saclay, 91190 Gif-sur-Yvette, France \\ ${ }^{6}$ European Southern Observatory, Alonso de Cordova 3107, Vitacura, Santiago, Chile \\ ${ }^{7}$ Atmospheric, Oceanic and Planetary Physics, Department of Physics, Clarendon Laboratory, \\ University of Oxford, Parks Road, Oxford, OX1 3PU, UK \\ ${ }^{8}$ Southwest Research Institute, 1050 Walnut St., Boulder, CO 80302, USA \\ ${ }^{9}$ NASA Headquarters, 300 E St. SW, Washington, DC 20546, USA \\ ${ }^{10}$ Boston University, Department of Astronomy and Center for Space Physics, Boston \\ University, 725 Commonwealth Avenue, Boston, MA 02215 \\ ${ }^{11}$ Space Telescope Science Institute, 3700 San Martin Dr., Baltimore, MD \\ 21218, USA \\ *Author to whom correspondence should be sent.
}

The Little Red Spot (LRS) in Jupiter's atmosphere was investigated in unprecedented detail by the New Horizons spacecraft together with the Hubble Space Telescope (HST) and the Very Large Telescope (VLT). The LRS and the larger Great Red Spot (GRS) of Jupiter are the largest known atmospheric storms in the solar system. Originally a white oval, the LRS formed from the mergers of three smaller storms in 1998 and 2000 and became as red as the GRS between 2005 and 2006. Here we show that circulation and wind speeds in the LRS have increased substantially since the Voyager and Galileo eras when the oval was white. The maximum tangential velocity of the LRS is now $172+/-18 \mathrm{~m} / \mathrm{s}$, close to the highest values ever seen in the GRS, which has also evolved both in size and maximum wind speed. The cloud top altitudes of the GRS and LRS are similar, both storms extending much higher in the atmosphere than other Jovian anticyclonic systems. The similarities in wind speeds, cloud morphology, and coloring suggest a common dynamical mechanism explains the reddening of the two largest anticyclonic systems on Jupiter. These storms will not be observed again from close range until at least 2016. 
Send Editorial Correspondence and Proofs to:

Andrew F. Cheng

Applied Physics Laboratory

11100 Johns Hopkins Rd

Laurel, MD 20723

Telephone: 240-228-5415

E-Mail: andrew.cheng@jhuapl.edu

Keywords: Jupiter, convection, hydrodynamics, atmospheres, storms 
Contact information:

Andrew F. Cheng

Applied Physics Laboratory

11100 Johns Hopkins Rd

Laurel, MD 20723

Telephone: 240-228-5415

E-Mail: andrew.cheng@,jhuapl.edu

A. F. Cheng, Johns Hopkins Applied Physics Laboratory, 11100 Johns Hopkins Rd., Laurel, MD 20723, USA. andrew.cheng@jhuapl.edu 240-228-5415

A. A. Simon-Miller, NASA/GSFC, Code 693, Greenbelt, MD 20771, USA.

Amy.Simon@nasa.gov 301-286-6738

H. A. Weaver, Johns Hopkins Applied Physics Laboratory, 11100 Johns Hopkins Rd., Laurel, MD 20723, USA. Hal.Weaver@jhuapl.edu 240-228-8078

K. H. Baines, G. S. Orton, P. A. Yanamandra-Fisher, Jet Propulsion Laboratory, 4800 Oak

Grove Dr., Pasadena, Calif. 91109, USA. kbaines@mail.jpl.nasa.gov 818-354-0481

go@scn.jpl.nasa.gov 818-354-246ㅜ padma@scn.jpl.nasa.gov 818-354-2321

O. Mousis, Institut UTINAM, CNRS-UMR 6213, Université de Franche-Comté, France.

olivier.mousis@obs-besancon.fr +33 381666921

E. Pantin, Centre d'Etudes Atomique CEA/SAp, Saclay, 91190 Gif-sur-Yvette, France. epantin@cea.fr +33169086577

L. Vanzi, European Southern Observatory, Alonso de Cordova 3107, Vitacura, Santiago, Chile. lvanzi@eso.org +56 55435311

L. N. Fletcher, Atmospheric, Oceanic and Planetary Physics, Department of Physics, Clarendon Laboratory, University of Oxford, Parks Road, Oxford, OX1 3PU, UK. fletcher@atm.ox.ac.uk +4401865272895

J. R. Spencer, Southwest Research Institute, 1050 Walnut St., Boulder, CO 80302, USA. spencer@,boulder.swri.edu 303-546-9674

S. A. Stern, NASA Headquarters, 300 E St. SW, Washington, DC 20546, USA.

alan.stern@nasa.gov 202-358-3889 
This paper discusses images of Jupiter's Little Red Spot (LRS) acquired by the LOng Range Reconnaissance Imager (LORRI) on 27 Feb 2007, shortly before the time of Jupiter closest approach by the New Horizons spacecraft (Fountain et al. 2007), which is on its way to a Pluto encounter in July 2015 (Stern et al. 2007), LORRI is a narrow angle telescope, with a 20.8-cm diameter primary mirror, a focal length of $263 \mathrm{~cm}$, and a 1024x1024 pixel, charge-coupled device detector (Cheng et al. 2007). LORRI's panchromatic (350 - $850 \mathrm{~nm}$ ) images were supported by contemporaneous images acquired by the Hubble Space Telescope (HST) at visible wavelengths and by the ESO Very Large Telescope (VLT) at mid-infrared wavelengths.

The LRS, about the size of the Earth, is an anticyclonic oval (counterclockwise rotation in the Southern Hemisphere). The LRS formed from the merger of three smaller anticyclonic ovals which appeared to form in the late 1930s (Peek 1958) and which were observed by the Voyager spacecraft in 1979, along with intervening cyclonic cells that helped to maintain their separation (Beebe et al. 1989). In 1998, two of the ovals merged, followed by a merger of the remaining two in 2000 (Sanchez-Lavega et al. 1999 \& 2001). In late 2005, the sole surviving oval became as red as the GRS.

How and why these giant storms form, and why they can persist for long times - since before 1879 in the case of the GRS (Peek 1958, Beebe et al. 1989) - remain mysteries. Also much debated are mechanisms for reddening of these storms, which may result from upwelling of material from depth (Taylor et al. 2004, West et al. 2004). Hence the recent formation of the LRS, which had consumed two other, smaller ovals and then became red, is of great interest, particularly in comparison with the GRS. Both the Voyager and the Galileo spacecraft at Jupiter mapped wind fields in the GRS and in the largest of the predecessor ovals, Oval BC, finding that these storms had similar dynamical structure (Mitchell et al. 1981, Simon et al. 1998, Vasavada et al. 1998, Simon-Miller et al. 2006).

Cloud-tracked wind measurements using successive image pairs were made for Oval BC, from Voyager data (from 1979) and Galileo data (1997 and 2000). The maximum tangential velocity was found to be a constant $120+/-5 \mathrm{~m} / \mathrm{s}$, despite size variations from 1979 to 1997 (SimonMiller et al. 2002). After the final merger in 2000, Cassini captured a single image pair of the still white oval that became the LRS, and maximum velocities were found to be $\sim 165+/-45 \mathrm{~m} / \mathrm{s}$, suggesting a possible velocity increase (Simon-Miller et al. 2006). After the LRS first became red, the highest available spatial resolution HST images $(\sim 75 \mathrm{~km} /$ pixel $)$ were used to infer that the maximum wind speed had increased to $180 \mathrm{~m} / \mathrm{s}$, but with large uncertainties of $70 \mathrm{~m} / \mathrm{s}$.

The next opportunity to obtain a high resolution wind field map of the LRS came with the New Horizons Jupiter encounter in 2007, which was the last such opportunity until the JUNO mission arrives in 2016, although JUNO does not plan to obtain velocity data. To search for changes in the wind field associated with the color change in the LRS, LORRI obtained a pair of $2 \times 2$ image mosaics from a distance of $\sim 33$ Jupiter radii, separated by a 30 -minute time interval. LORRI was not used to measure wind fields in the GRS because of limited data volume. The maximum spatial resolution in the LRS was $14.4 \mathrm{~km} /$ pixel, for a single-pixel measurement uncertainty of 8 $\mathrm{m} / \mathrm{s}$. As shown in Figure 1a, the highest measured wind speed in the LRS is $172+/-18 \mathrm{~m} / \mathrm{s}$, consistent with previous Cassini and HST data (Simon-Miller et al. 2006), but with significantly smaller error bars, indicating an increased wind speed relative to Galileo and Voyager data (see Table 1). All of these wind field measurements were made with a consistent methodology, so comparisons are valid.
Deleted:

Deleted: will be 
Just as for the GRS, there is a counterclockwise flow within the LRS (Fig. 1a) and a central region (extending about $1^{\circ}$ in latitude and $1.5^{\circ}$ in longitude) with brighter clouds, where the wind speed is below the measurement uncertainty of $\sim 18 \mathrm{~m} / \mathrm{s}$ (Fig. 1b). The flow speed is greatest toward the outer edge of the LRS where the rotation period is $\sim 35$ hours. With a peak velocity of $172 \mathrm{~m} / \mathrm{s}$, the maximum Rossby number along the major axis is $\mathrm{R}_{\mathrm{o}}=0.41$ (formulae are shown in the Appendix). This is higher than was found for Oval BC from Voyager data (Mitchell et al. 1981), and the corresponding relative vorticity along the major axis at the maximum velocity contour has increased by $\sim 16 \%$ and has doubled along the minor axis (Table 1 ). A comparable analysis of Galileo velocity vectors for the GRS shows a slightly lower maximum Rossby number, although the relative vorticity along the semi-minor axis has increased threefold from Voyager to Galileo; the GRS also increased in maximum tangential velocity to $170+/-12 \mathrm{~m} / \mathrm{s}$ and decreased in longitudinal extent (Simon-Miller et al. 1998, Simon-Miller et al. 2002, Sada et al. 1996).

The LRS exhibits much lower Rossby numbers along its northern and southern sides than near the more sharply curved eastern and western ends, indicating essentially geostrophic flow (an approximate balance between Coriolis force and pressure gradient) except near these ends. The overall roundness of the flow, which bears upon dynamical balance, is measured by eccentricity. The Rossby numbers shown in Table 1 are close to the maximum values attained in the flows and indicate the greatest deviations from geostrophic balance. This dynamical structure in the LRS after the color change was also found both in the precursor Oval BC and in the GRS (Mitchell et al. 1981). Although the overall dynamical structure was maintained before and after the color change, the circulation around the maximum velocity contour (line integral of tangential velocity), which measures the strength of a vortex, increased significantly for the LRS versus the white oval BC. The circulation in the GRS also increased between the Voyager and Galileo eras (Table 1).

For both the GRS and LRS, the relative vorticity along the major axis is much higher than the ambient zonal wind shear $\sim 10^{-5} \mathrm{~s}^{-1}$ (Mitchell et al., 1981 and Figure 1c), an indication of the strength of these storms. The background winds, derived by tracking small cloud features with HST data from 26 Feb. 2007, show that westward jets north of each storm's center are deflected to the north. The zonal winds were obtained from ten-hour separated HST image pairs. Uncertainties range from $\sim 3$ to $11 \mathrm{~m} / \mathrm{s}$ depending on the latitude and the number of features that could be tracked within a $0.5 \mathrm{deg}$ latitude bin. For the GRS, small eddies occasionally appear on the westward jet, approach from the east and are pulled into the interior, possibly adding to the unknown energetics (Beebe et al. 1989, Mitchell et al. 1981). This has not been directly observed for the LRS, although the morphologies of clouds around its edges indicate interaction with its surroundings. In most regions surrounding the LRS, wind speeds are below $30 \mathrm{~m} / \mathrm{s}$, too low to be measured accurately from the LORRI image mosaic pairs. However, anticyclonic flow is suggested in the small oval southeast of the LRS, near $215^{\circ} \mathrm{W}, 40^{\circ} \mathrm{S}$ (Fig. 1a), and in the small cloud core near $229^{\circ} \mathrm{W}, 31^{\circ} \mathrm{S}$,

Similar to the GRS(Beebe et al. 1989, Simon-Miller et al. 2002, Vincent et al. 2000), the LRS extends vertically into the stratosphere, as indicated by its brightness in methane gas absorption wavelengths and darkness at ultraviolet wavelengths, Figure 2 shows that the LRS extends to higher altitudes than the smaller anticyclone at $40^{\circ} \mathrm{S}$, as indicated by the absence of the smaller storm at $0.26 \mu \mathrm{m}$ and its fainter appearance in the $0.89-\mu \mathrm{m}$ maps.

Deleted: LRS
Deleted: GRS
Formatted: Superscript
Formatted: Superscript
Deleted: zonal

Deleted: 29

Deleted: (Fig. 1a)

Deleted: (Beebe et al. 1989, SimonMiller et al. 2002, Vincent et al. 2000)

Deleted: given 
Just north of the LRS at the time of the New Horizons Jupiter encounter, there was a South Tropical Disturbance (STrD), a portion of which is seen in Figure 1a. This STrD formed in late 2006 (Rogers, 2007) and is comparable in size to the GRS (Figure 1c). The observed wind fields within the observed portion of the STrD (Figure 1a) are consistent with anticyclonic flow. This flow is notably less organized than within the LRS, with maximum speeds on the order of 50 $\mathrm{m} / \mathrm{s}$. Figure $1 \mathrm{~b}$ shows that colors within the STrD are similar to those within the small oval southeast of the LRS, indicating similar tropospheric clouds and haze color.

Ground-based mid-IR images taken to support the LORRI observations show similarities between the vertical structure and dynamics of the GRS and LRS (Fig. 2). These observations were acquired using the VISIR camera/spectrometer (LaGage et al. 2004) at the VLT UT-3 telescope (Melipal) on 28 February 2007. The thermal images which included the LRS covered most of Jupiter's southern hemisphere, enabling the assignment of latitudes, longitudes and emission angles to each pixel. In order to combine the information from the images at different wavelengths, the images were reformatted as cylindrical maps with consistent longitude and latitude grids. Observations were made with standard rapid-frequency chopping against the sky 15 arc seconds to Jupiter's south and nodding further south for another chopped image pair in order to characterize accurately and subtract telluric sky emission from Jupiter's much fainter emission. Although the VLT images were taken one day later than the LORRI images in Figure 1a, no large-scale changes in structure or dynamics are expected based upon previous observations of Jupiter's atmosphere.

Temperatures were derived from images taken in narrow-band filters with central wavelengths of $13.04,17.65,18.72$ and $19.50 \mu \mathrm{m}$ which are sensitive to thermal emission from a portion of the spectrum dominated by collision-induced $\mathrm{H}_{2}$ absorption (e.g. Flasar et al. 2004). Because $\mathrm{H}_{2}$ is well-mixed in Jupiter's atmosphere, variations of the upwelling radiance can be used to determine variations of the atmospheric temperatures between 100 and 400 mbar total pressure. Temperatures were retrieved by simultaneously fitting the radiances in all the filters cited above, obtaining estimates of the relative variation of $T(p)$ across the LRS and its surroundings. The reference Jovian atmosphere profiles were from Galileo Probe data (smoothed to remove wave signatures), and the radiance of the images was adjusted for consistency with the results of the Cassini Composite Infrared Spectrometer (CIRS) at Jupiter (Flasar et al. 2004). The estimated error of temperature retrieval from the radiative transfer modeling is about $2.2 \mathrm{~K}$ at 500 mbar and $2.9 \mathrm{~K}$ at $100 \mathrm{mbar}$ in each $\sim 1^{\circ} \times 1^{\circ}$ spatial resolution element. An equilibrium ortho/para hydrogen ratio was assumed. An adaptation of the Oxford-based Nemesis optimal estimation retrieval algorithm (Parrish 2004) was used in these retrievals. Gases included in the reference model (Kunde et al. 2004, Parrish 2004, Wong et al. 2004) were hydrogen, helium, methane, $\mathrm{CH}_{3} \mathrm{D}$, ammonia, ethane and acetylene. Similarly filtered radiances at $8.59 \mu \mathrm{m}$ were used to assess the opacity of a 600-mbar to 1-bar cloud and account for its influence on upwelling radiances at the longer wavelengths.

The temperatures derived from these thermal images show that the LRS (Fig. 3) has a cold central region, roughly coincident with the visible edges of the oval at all levels of the upper troposphere between 200 and 400 mbar. This is similar to the GRS (Simon-Miller et al. 2002), and both giant anticyclones also have collars along their peripheries which are warmer than the surrounding atmosphere. The GRS has evidence for inhomogeneous distributions of ammonia and clouds near 300-600 mbar (Fig. 2). Similar to the GRS, the cold interior temperatures seen in Fig. 3 clearly identify the LRS as an anticyclone, with its coolest region roughly coincident with
Deleted: 3

Deleted: a distinctly warmer collar than the LRS, as well as 
the location of the visible cloud oval. The thermal images also clearly identify the small oval at $40^{\circ} \mathrm{S}$ (marked by an arrow in Fig. 2) as a lower-temperature anticyclone. The thermal images also confirm the anticyclonic nature of the STrD just north of the LRS. The temperatures are consistent with an interpretation that gas is upwelling in both the LRS and the nearby small oval which expands and cools as it rises, similar to the GRS (Simon-Miller et al. 2002). This gas subsides everywhere exterior to the boundary, warming the atmosphere.

Both the GRS and the LRS show enhanced emission along with southern portions of their collars (Fig. 2 and Fig. 3 for the LRS) Historical data indicate that this is a recurring feature for the GRS (Simon-Miller et al. 2002), although not for the LRS_precursor ovals. Voyager and Cassini observations of the GRS, showing the enhanced southern temperatures, were interpreted as evidence of a north-to-south tilt to the storm (the visible cloud deck is at lower altitude in the south) or a spiral-shaped flow (Simon-Miller et al. 2002). The similar dynamical structures of the GRS and LRS argue that the enhanced southern temperature for the LRS also reflects a tilt or spiral form.

However, an alternative interpretation is that the warming along the southern edge of the LRS may be temporary and could be the result of downwelling forced by an interaction between the LRS and the STrD which is just to the north. Figure 3 shows a prominent warm region south of the LRS, with a size and temperature contrast to the ambient at least comparable to those of the LRS. Although there is no clear expression of this thermal feature in visible cloud morphology, evidence of cyclonic flow is seen in Figure 1a, where the wind vectors point systematically to the west near $40^{\circ} \mathrm{S}$, at speeds similar to those within the LRS. If indeed there is significant interaction between the STrD, the LRS, and the warm cyclonic region to the south, this interacting complex would dwarf the GRS.

A complication is that the GRS images in Figure 2 at 8.59 and $10.77 \mu \mathrm{m}$, sensitive not only to temperatures but also to $\sim 600$-mbar cloud opacity and 300-mbar ammonia gas absorption, respectively, show evidence for inhomogeneous distributions of those quantities. Specifically, there are patchy brightness enhancements in the southern portion of the GRS at 8.59 and at 10.77 $\mu \mathrm{m}$, although similar enhancements are not evident within the LRS. In addition, as can be seen in the 8.59 and $10.77 \mu \mathrm{m}$ LRS images in Figure 2, as well as the thermal maps of Figure 3, there is a warm ring extending completely around the LRS, separating it from the cold STrD. The thermal observations support the suggestion of an interaction between the LRS and the STrD, in which case the warm cyclonic region to the south is a distinct phenomenon from the warmer southern collar seen at the GRS. The latter may be a durable structural feature (Simon-Miller et al. 2002), which may also help to explain the southern brightness enhancements at 8.59 and $10.77 \mu \mathrm{m}$ within the GRS. Existing axially symmetric models of these anticyclonic vortices do not address these complications.

The 2007 New Horizons observations demonstrate convincingly that the maximum wind speeds and circulation in the LRS are dramatically increased over those seen in 1997, when the oval was white. With a north-to-south tilt and/or spiral form, the increased circulation would imply an increased vertical transport, which may be associated with the color change. However, the increase from Voyager to Galileo in the circulation of the GRS, which also has a north-south tilt and/or spiral form, was not accompanied by a color change. Perhaps in the case of the GRS, which was already red, the rate of vertical transport was already sufficiently large that a further increase would not change the color; whereas the LRS may have crossed a threshold where the increased vertical transport led to a color change. The precise temporal relationship between the 
LRS color change and the increase in circulation remains unclear, because of the long time intervals between spacecraft observations of high resolution wind fields. Both the temperature and velocity structures of the LRS remain similar to those of the GRS, although the LRS at the time of the New Horizons Jupiter encounter may have been influenced by the nearby STrD. However, the GRS has steadily become rounder and decreased in longitudinal extent since Voyager (Beebe et al. 1989, Simon-Miller et al. 2002). The decrease in size partly compensated for an increase in maximum wind speed, so the circulation in the GRS underwent a relatively minor increase from Voyager to Galileo, compared to the later increase observed for the LRS. It is not clear that the GRS will remain indefinitely as the largest and strongest storm on Jupiter in terms of circulation. It also remains unclear whether the evolution of the GRS and LRS is an aspect of climate cycling on Jupiter, whereby these storms may disappear in the future (Marcus 2004), and why large, long-lived anticyclones are more prevalent in the southern hemisphere than in the north. Further work is indicated in modeling these giant anticyclones in order to provide consistency with these New Horizons images, together with historical observations in the visible and the advent of simultaneous constraints provided by mid-IR observations with spatial resolutions of $2000 \mathrm{~km}$ or better.

Beebe, R. F. and L.A. Youngblood. Nature, 280,771-772 (1979)

Beebe, R. F. et al. Time Variable Phenomena in the Jovian System (1989)

Cheng, A. F. et al. Space Science Reviews, submitted (2007), preprint available at http://arxiv.org/abs/0709.4278

Flasar, F. M. et al. 2004. Nature. 427, 132 - 135.

Fountain, G. et al. Space Science Reviews, submitted (2007), preprint available at http://arxiv.org/abs/0709.4288

Kunde, V. G., et al. 2004. Science 305, 1582-1587.

LaGage, P. O. et al. The Messenger. 117, 112 (2004)

Marcus, P. Nature, 428, 828 (2004)

Mitchell, J. L. et al. JGR, 86, 8751-8757 (1981)

Parrish, P. D., 2004. Global retrievals of upper tropospheric phosphine from the Cassini/CIRS Jupiter encounter. Ph.D. thesis, University of Oxford.

Peek, B. M. The Planet Jupiter (1958)

Rogers, J. H. J Br. Astron. Assoc., 117, 113-115 (2007)

Sada, P. V. et al. Icarus, 119, 311-335 (1996)

Sanchez-Lavega, A. et al. Icarus, 149, 491-495 (2001)

Sanchez-Lavega, A. et al., Icarus, 142, 116-124 (1999)

Simon, A. A. et al. Icarus, 135, 220-229 (1998)

Simon-Miller, A. A. et al. Icarus, 158, 249-266 (2002)

Simon-Miller, A. A. et al. Icarus, 185, 558-562 (2006)

Stern, S. A. et al. Space Science Reviews, submitted (2007), preprint available at http://arxiv.org/abs/0709.4417

Taylor, F. W. et al. 2004 Chapter 4 of Jupiter: the Planet, Satellites and Magnetosphere (eds: Bagenal, Dowling, McKinnon)

Vasavada, A. R. et al. Icarus, 135, 265-275 (1998)

Vincent, M. B. et al. Icarus 143, 189-204 (2000)

West, R. A. et al. 2004 Chapter 5 of Jupiter: the Planet, Satellites and Magnetosphere (eds: Bagenal, Dowling, McKinnon) 
Wong, M. H. et al. Plan. \& Space Sci. 52, 385-395 (2004)

Supplementary Information is linked to the online version of this paper: blink movie

Acknowledgements We thank the entire New Horizons mission team and our colleagues on the New Horizons science team. New Horizons is funded by NASA, whose financial support we gratefully acknowledge. We thank the Space Telescope Science Institute, which is operated by the Association of Universities for Research in Astronomy, Inc., for Director's Discretionary time granted to the Hubble Heritage Project, PI K. Noll. We also thank I. Tsavaris for HST wind field measurements. We acknowledge the European Southern Observatory staff at the Very

Large Telescope, as well as the Caltech Summer Undergraduate Research Fellows E. Edkins and J. Joels at JPL, for help in rapid reduction of the VLT data.

Author Information Correspondence and requests for materials should be addressed to A. F. Cheng at andrew.cheng@jhuapl.edu 
Table 1.

\begin{tabular}{|c|c|c|c|c|c|c|c|c|}
\hline & $\begin{array}{c}\text { Maximum } \\
\text { Tangential } \\
\text { Velocity } \\
(\mathrm{m} / \mathrm{s})\end{array}$ & $\begin{array}{l}{ }^{1} \text { Semi- } \\
\text { major } \\
\text { Axis, } a \\
\left(\times 10^{6} \mathrm{~m}\right)\end{array}$ & $\begin{array}{l}{ }^{1} \text { Semi- } \\
\text { minor } \\
\text { Axis, } b \\
\left(\times 10^{6} \mathrm{~m}\right)\end{array}$ & $\begin{array}{c}\text { Eccentri } \\
\text {-city }\end{array}$ & $\begin{array}{l}{ }^{2} \text { Rossby } \\
\text { Number }\end{array}$ & $\begin{array}{c}{ }^{2} \text { Relative } \\
\text { Vorticity, } \\
\text { along } a \\
\left(\times 10^{-5} \mathrm{~s}^{-1}\right)\end{array}$ & $\begin{array}{l}{ }^{3} \text { Relative } \\
\text { Vorticity, } \\
\text { along } b \\
\left(\times 10^{-5} \mathrm{~s}^{-1}\right)\end{array}$ & $\begin{array}{l}{ }^{4} \text { Circul- } \\
\text { ation } \\
\left(\mathrm{x} 10^{8}\right. \\
\left.\mathrm{m}^{2} / \mathrm{s}\right)\end{array}$ \\
\hline \multicolumn{9}{|c|}{ GRS } \\
\hline $\begin{array}{c}\text { Voyager, } \\
1979\end{array}$ & $\begin{array}{c}110+/- \\
12\end{array}$ & 10.5 & 4.85 & .887 & .40 & 5.4 & 0.5 & 59.8 \\
\hline $\begin{array}{c}\text { Galileo, } \\
2000\end{array}$ & $\begin{array}{c}170+/- \\
12\end{array}$ & 8.17 & 5.83 & .700 & .30 & 4.1 & 1.5 & 75.3 \\
\hline \multicolumn{9}{|c|}{ LRS/Oval BC } \\
\hline $\begin{array}{c}\text { Voyager, } \\
1979\end{array}$ & $120+/-5$ & 4.89 & 2.93 & .801 & .36 & 6.8 & 1.5 & 29.9 \\
\hline $\begin{array}{c}\text { Galileo, } \\
1997\end{array}$ & $\begin{array}{c}120+/- \\
20\end{array}$ & 3.80 & 2.92 & .640 & .28 & 5.3 & 2.4 & 25.4 \\
\hline $\begin{array}{c}\text { New } \\
\text { Horizons, } \\
2007\end{array}$ & $\begin{array}{c}172+/- \\
18\end{array}$ & 3.93 & 2.92 & .669 & .41 & 7.9 & 3.2 & 37.2 \\
\hline
\end{tabular}

1. Dimensions of the maximum velocity contour.

2. Calculated at the maximum velocity contour, along the semi-major axis

3. Calculated at the maximum velocity contour, along the semi-minor axis.

4. Calculated on the maximum velocity contour. 


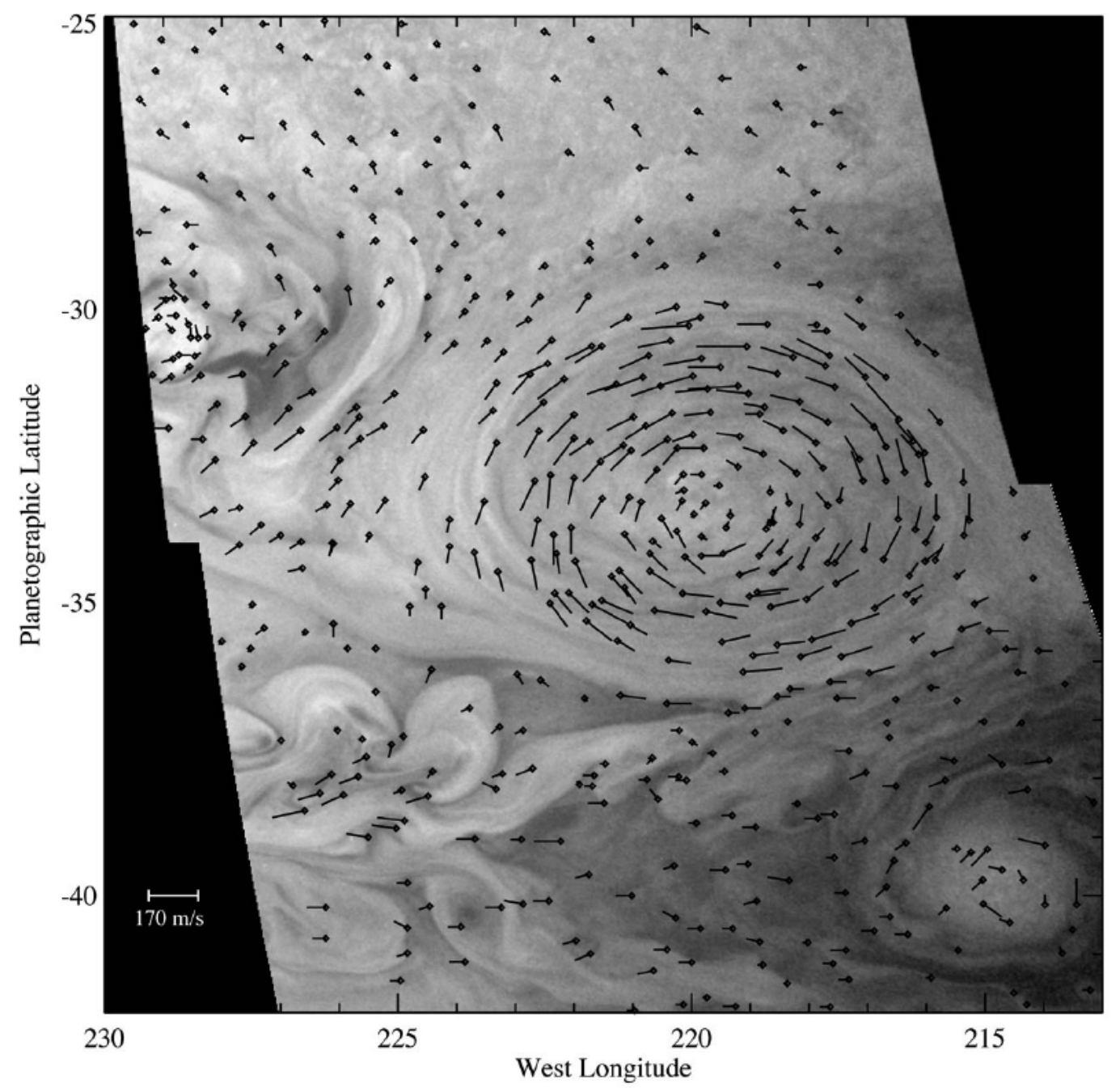

Figure 1. (a) Wind vectors measured from the LORRI frames, plotted on the first time step. Wind vectors have been slightly exaggerated for clarity and diamond symbols indicate the starting point for the vector. The LRS is the counterclockwise circulating oval to the right of center. An STrD is seen at the top, north of $29^{\circ} \mathrm{S}$. Winds to the west are observed near $40^{\circ} \mathrm{S}$ outside of the small oval at lower right. 


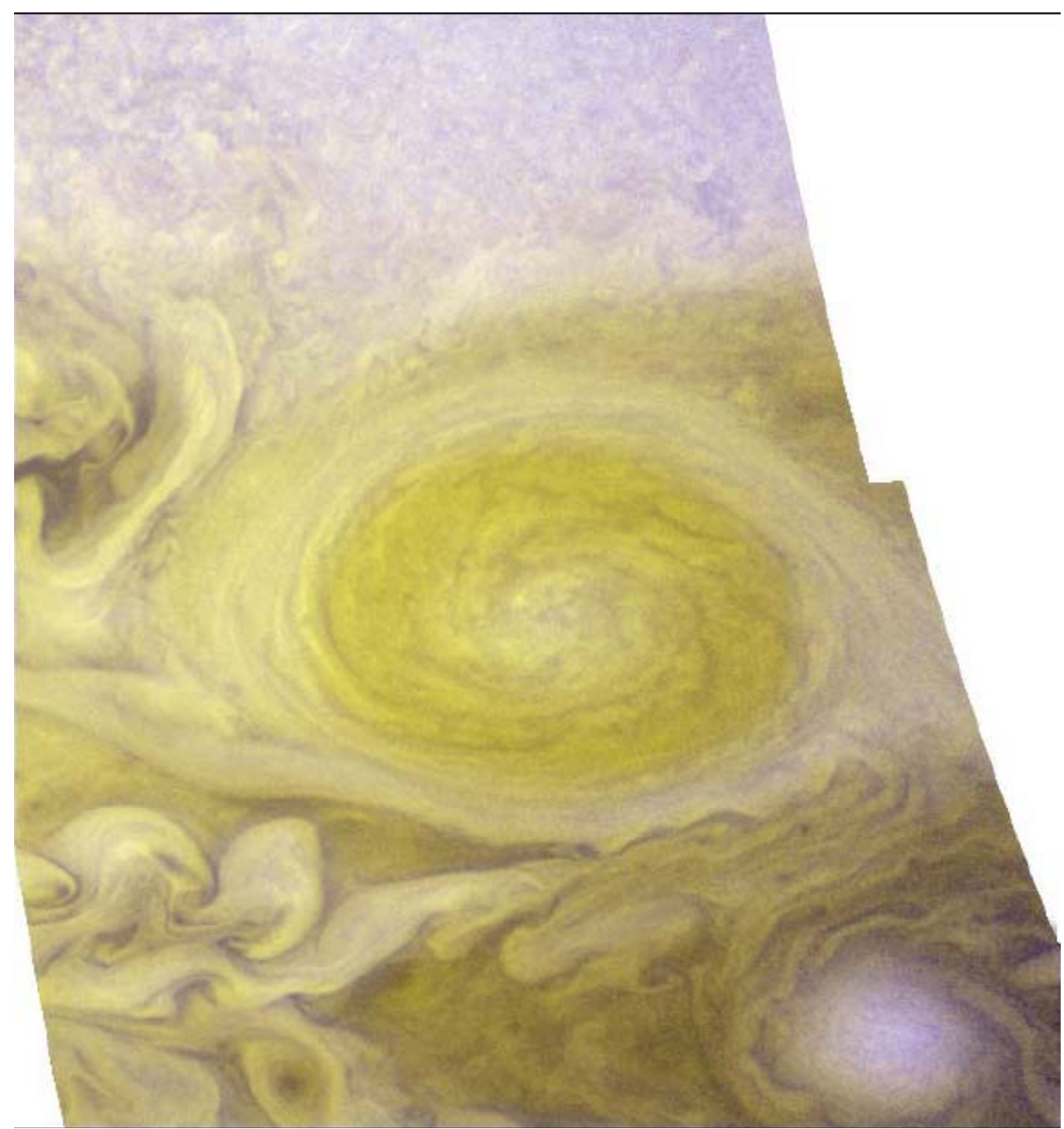

Figure 1. (b) Quasi true-color view of the LRS, generated using a LORRI mosaic in the red and green channels and an HST 410-nm map in the blue channel. The LRS appears with distinctly redder color than the STrD to the north or the small oval to the southeast.

\begin{tabular}{|l|}
\hline Deleted: STrD \\
\hline Deleted: a \\
\hline Deleted: from \\
\hline Deleted: LRS \\
\hline Deleted: .
\end{tabular}




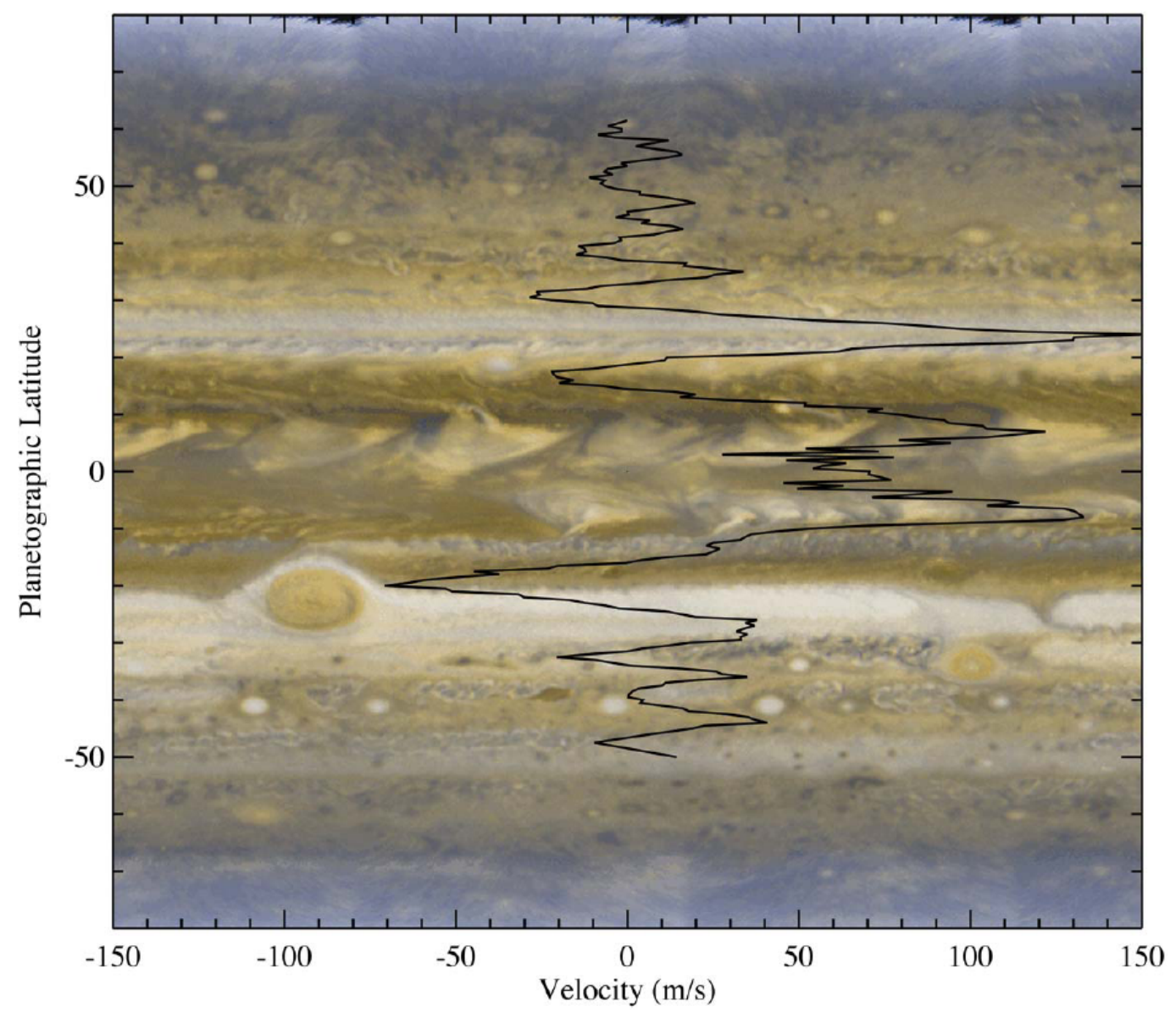

Figure 1. (c) HST mosaic from Feb 26, 2007 showing the GRS and LRS regions, with an average zonal wind field overplotted ${ }_{k}$ Color is generated using a 410-nm map (blue) and 673-nm map (red), with a composite of the two in the green channel. The GRS is at the left near $20^{\circ} \mathrm{S}$, and the LRS with the STrD to the north is seen at the right. The STrD is darker than other whitish clouds Deleted: The GRS is at the left near $20^{\circ} \mathrm{S}$, and the LRS with the darker STrD in its latitude band which overlaps that of the GRS. 
LRS
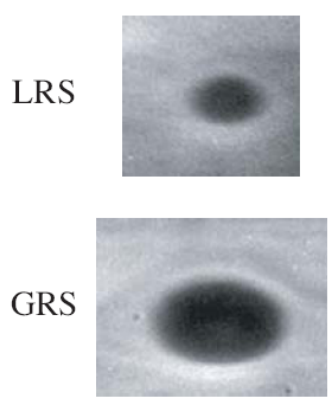

0.26
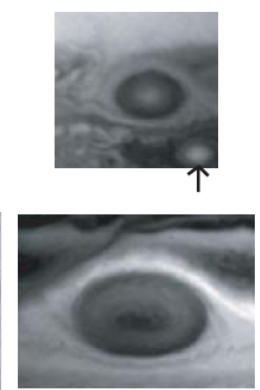

0.41
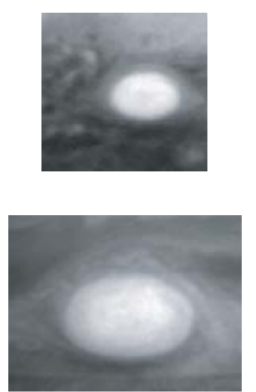

0.89
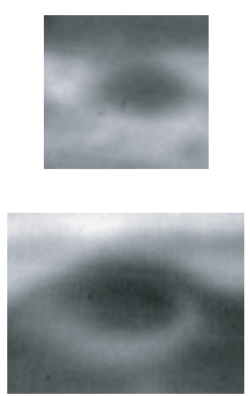

8.59
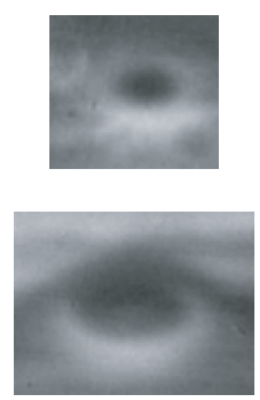

10.77

Figure 2. A multi-wavelength comparison of the LRS (top) and GRS (bottom). Images are not to scale, and all wavelengths are listed in microns. The $0.26,0.41$ and 0.89 -micron images are from HST, acquired on Feb. 26, 2007. The 8.59 and 10.77-micron images were acquired on Feb. 28 (LRS) and Mar. 1, 2007 (GRS) at the VLT. The 0.26-micron filter is sensitive to stratosphere haze, 0.41 microns to troposphere clouds and haze color, 0.89 microns to upper troposphere cloud and haze opacity, 8.59 microns to troposphere cloud opacity and temperature, and 10.77 microns to troposphere temperature and ammonia gas. Black arrow marks a small oval southeast of the LRS. The southern portion of the GRS is brighter at 8.59 and $10.77 \mu \mathrm{m}$. 
a

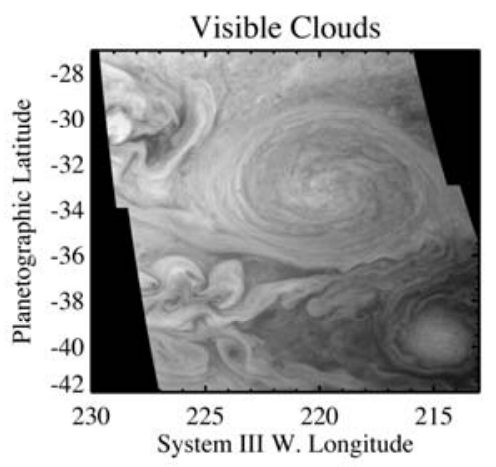

C

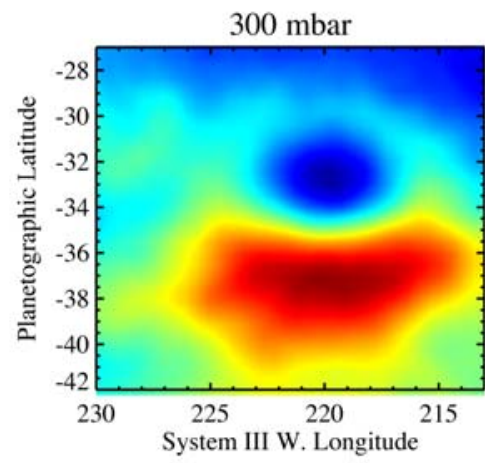

b

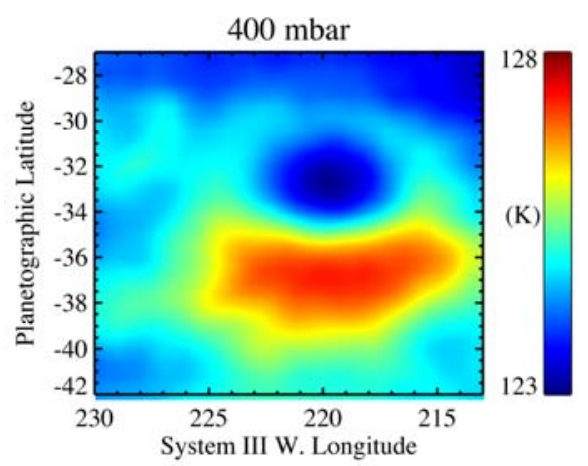

d
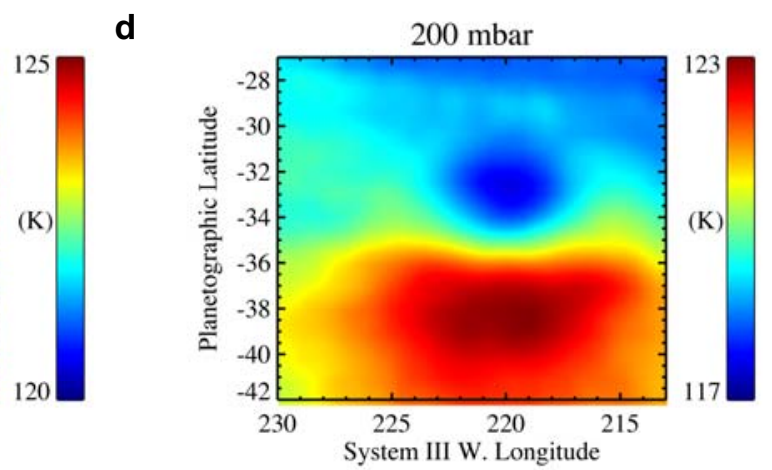

Figure 3. (a) visible image from New Horizons. Temperature maps on constant pressure surfaces at 400 mbar (panel b), 300 mbar (panel c), and 200 mbar (panel d) from inversion of images of Jupiter's thermal radiances taken through $\sim 1-\mu \mathrm{m}$ wide filters centered at 13.04, 17.65, 18.72, and $19.50 \mu \mathrm{m}$ which are primarily sensitive to temperatures in the $200-400$ mbar pressure range, unlike the mid-infrared images shown in Fig. 2. The spatial resolution is equivalent to $1.5^{\circ}$ in longitude and $1.8^{\circ}$ in latitude. The LRS and small oval are cold anticyclones, as is the STrD. A warm, cyclonic region is seen south of the LRS. 


\section{Appendices}

Determining Eccentricity, Relative Vorticity and Rossby Number

Ellipse eccentricity, $\varepsilon$, is defined as:

$$
\varepsilon=\left(1-(b / a)^{2}\right)^{1 / 2}
$$

where $a$ is the semi-major axis, and $b$ is the semi-minor axis.

For all vorticity and Rossby number calculations, we follow the derivations of Mitchell, et al. 1981 (8). This Mitchell et al. model is used to obtain more accurate and reliable estimates of flow quantities like vorticity from observational data which are subject to limitations of sampling and measurement error. The time spans over which comparisons are made in Table 1 are much longer than dynamical or thermal timescales, so it is reasonable to use this model to make flow measurements. Thus, relative vorticity, $\zeta$, is defined as:

$$
\zeta=v_{T} a /\left(b^{2} \eta^{3}\right)+\eta d v_{T} / d a
$$

where $v_{T}$ is the tangential velocity, and $\eta$ is defined by:

$$
\eta=\left[\left(\cos ^{2} \theta+(a / b)^{4} \sin ^{2} \theta\right) /\left(\cos ^{2} \theta+(a / b)^{2} \sin ^{2} \theta\right)\right]^{1 / 2}
$$

where $\theta$ is the angle of the velocity point measured from east (along the semi-major axis) through north. Therefore, for $\theta=0^{\circ}, \eta=1$, and for $\theta=90^{\circ}, \eta=a / b$. For all Rossby number calculations in Table $1, \theta=0^{\circ}$ was used.

Finally, the maximum Rossby number, Ro, is calculated using the following formula:

$$
R o=v_{T}^{*} a /\left(b^{2} \eta^{3} f\right)
$$

where $f$ is the Coriolis parameter at each storm's central latitude, i.e., $13.5 \times 10^{-5} \mathrm{~s}^{-1}$ for the GRS and $19.2 \times 10^{-5} \mathrm{~s}^{-1}$ for the LRS.

The measured tangential wind speeds versus semi-major axis $a$ are fitted to an analytic function of the form 


$$
\begin{aligned}
& v_{T}(a)=K \varphi(y) \Phi(\alpha y)-B \\
& y=\beta\left(a-a_{0}\right) \\
& \varphi(y)=\frac{1}{\sqrt{2 \pi}} \exp \left(-\frac{y^{2}}{2}\right) \\
& \Phi(\alpha y)=\int_{-\infty}^{\alpha y} \varphi(t) d t
\end{aligned}
$$

with 5 constants. This form replaces the polynomial form of Mitchell et al. 1981 (8), as it is better suited to describing highly skewed velocity distributions which peak near the outer edges of the ovals. In the first equation, the three constants are $K$ (an overall scaling factor), $B$ (a constant bias), and $\alpha$ which controls the skewness. The second equation has another two constants, $\beta$ which is inversely related to the width of the peak, and $a_{0}$ which dominates the location of the peak. The function $\phi(y)$ is the standard normal distribution, and $\Phi(\alpha y)$ is the cumulative normal distribution evaluated at $\alpha y$. Here $a$ is as in Mitchell et al. (8) the semi-major axis in units of $10^{6} \mathrm{~m}$ and $v_{T}$ is likewise the tangential wind velocity in $\mathrm{m} / \mathrm{s}$.

The analytic function fit to the wind speed observations in the oval is used to evaluate the flow quantities vorticity, Rossby number, and circulation. This procedure is robust against the observational errors in individual wind speed measurements. 